\title{
Modelling of pneumatic muscle actuator using Hill's model with different approximations of static characteristics of artificial muscle
}

\author{
Ján Pitel ${ }^{1}, \mathrm{a}$ and Mária Tóthová ${ }^{1}$ \\ ${ }^{1}$ Department of Mathematics, Informatics and Cybernetics, Faculty of Manufacturing Technologies, Technical University of Košice, \\ Bayerova 1, 08001 Prešov, Slovakia
}

\begin{abstract}
For modelling and simulation of pneumatic muscle actuators the mathematical dependence of the muscle force on the muscle contraction at different pressures in the muscles is necessary to know. For this purpose the static characteristics of the pneumatic artificial muscle type FESTO MAS-20-250N used in the experiments were approximated. In the paper there are shown some simulation results of the pneumatic muscle actuator dynamics using modified Hill's muscle model, in which four different approximations of static characteristics of artificial muscle were used.
\end{abstract}

\section{Introduction}

Pneumatic muscle actuators for robotics and biomedical engineering applications [1,2] are usually designed as a kinematic structure, which corresponds to a one degreeof-freedom (DOF) system with one rotary axis [3]. These actuators are characterized by several appealing properties such as advantageous power/weight ratio, natural compliance, cleanness and structural simplicity. They consist of a pair of pneumatic artificial muscles (PAMs) arranged in antagonistic configuration (acting against themselves) in order to generate a retraction force or rotary movement (Figure 1) [4].

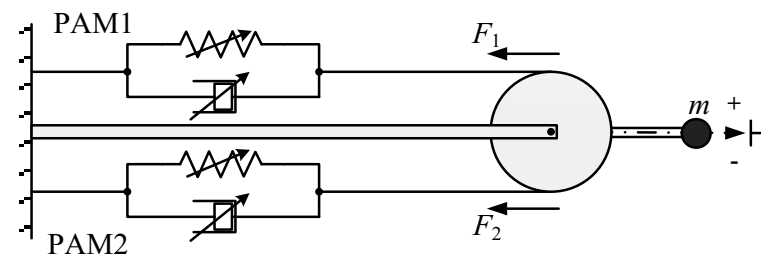

Figure 1. Model of antagonistic pneumatic muscle actuator using modified Hill's model

PAMs are made of rubber elastic tube with nonextensible braiding made of various materials (e.g. nylon), which defines an expansion in the context of increasing pressure. Depending on the actual construction of PAMs as well as operating condition, the highly nonlinear and also hysteretic properties may be more or less pronounced. Therefore it is necessary to use modeling for the better understanding the design parameters of PAMs in order to find dynamic muscle characteristics. These models are also important for simulation of the movement dynamics of the pneumatic muscle actuators and also for their control.

\footnotetext{
a Corresponding author: jan.pitel@tuke.sk
}

\section{Modified Hill's muscle model}

The modeling of PAMs as a main part of pneumatic muscle actuators was the subject of study from the very beginning of renewed interest about PAMs [5-9]. One of the simplest models of PAM is the simple geometric [10] and the advanced geometric [11] muscle model which dominate muscle modeling for simplicity and low demands on computing power. They depend on the geometrical properties of the muscle and physical processes running inside the muscle. The modified Hill's muscle model uses an engineering approach to muscle modeling, it is one of the oldest models and belongs to phenomenological models, that consists of a variable damper and a variable spring connected in parallel (Figure 1) [4]. Dynamic simulation model of antagonistic pneumatic muscle actuator using modified Hill's model was realized in Matlab/Simulink environment (Figure 2).

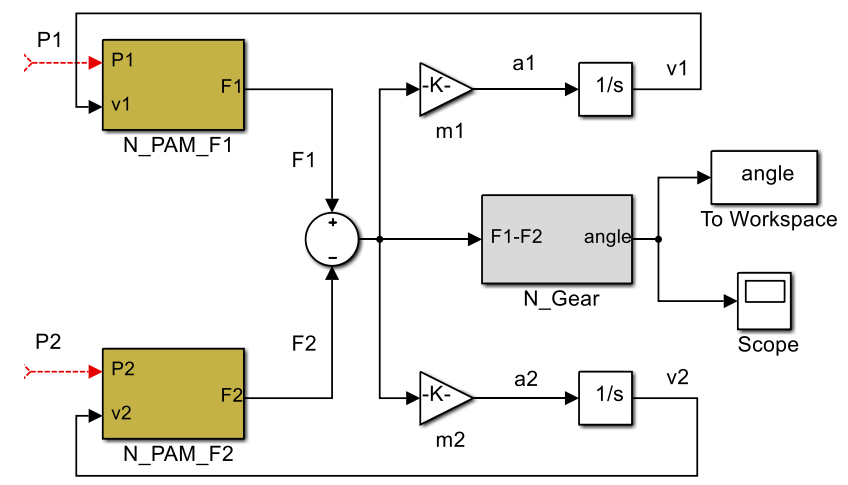

Figure 2. Simulation model of antagonistic pneumatic muscle actuator using modified Hill's model 
The dynamics of the PAM system consists of the series-parallel combination of standard mechanical components [12]. It can be described by the following nonlinear differential equation based on the Newton's second law:

$$
\ddot{y}=\frac{1}{m}\left[F_{E}-F_{s}(\kappa, P)-F_{d}(\dot{\kappa}, P)\right],
$$

where $m$ is the moving mass, $y$ is the muscle displacement $[\mathrm{m}], F_{s}(\kappa, P)$ is the nonlinear term representing a variable spring force, $F_{d}(\dot{\kappa}, P)$ is the nonlinear term representing a damper force, $F_{E}$ is the external force $[\mathrm{N}], P$ is the absolute muscle pressure $[\mathrm{Pa}]$ and $\kappa$ is the muscle contraction (defined as $\kappa=\kappa_{0}+y / l_{0}$, where $\kappa_{0}$ is the initial contraction [-] and $l_{0}$ is the initial muscle length $[\mathrm{m}]$.

The member $F_{E}$ (external force) represents all forces to which a muscle is subjected. It can be for example the gravitational force of a load or the frictional force. In case of use muscles antagonistic connection, it is the force of the other muscle.

The force of variable spring $F_{s}(\kappa, P)$ represents an active member in the modified Hill's muscle model and it is described in section 3 .

The force of damper $F_{d}(\dot{\kappa}, P)$ represents a passive member in the modified Hill's muscle model and it is a nonlinear (due to the multiplication) function of the velocity of muscle movement and the muscle pressure [12]:

$$
F_{d}(\dot{\kappa}, P)=R \cdot P \cdot \dot{\kappa},
$$

where $R$ is the damping coefficient $\left[\mathrm{m}^{2} \cdot \mathrm{s}\right], P$ is the absolute muscle pressure $[\mathrm{Pa}]$ and $\dot{\kappa}$ is the muscle velocity $\left[\mathrm{s}^{-1}\right]$.

Modified Hill's muscle model does not include internal structure and function of the muscle. PAM in this model generates two types of forces, active $F_{s}$ and passive $F_{d}$, which together form a resultant force of the muscle (Figure 3). The contractile member in Hill's muscle model is an active source of power which is controlled by the muscle pressure. Then the force of contractile element is a nonlinear function $(\mathrm{f}(\mathrm{u})$ in Figure 3) of two variables, contraction and pressure in the muscle [13].

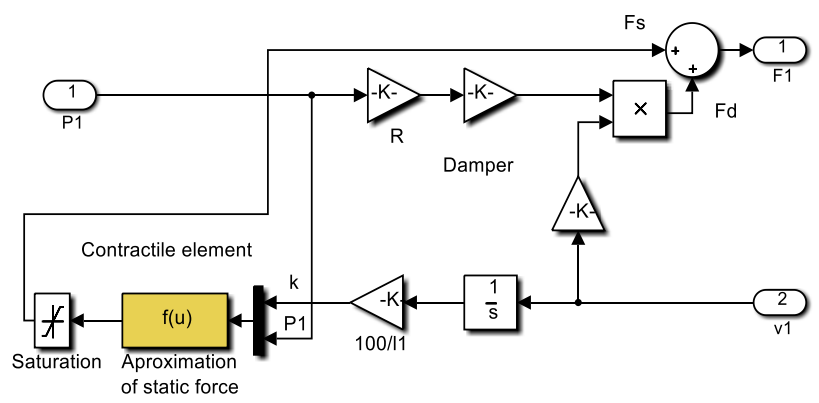

Figure 3. Subsystem N_PAM F of the muscle force nonlinearity

\section{Approximations of the PAM static characteristics}

The muscle force dependence on the muscle contraction and pressure in the muscle (Figure 4) [14] was approximated using available software tools as Matlab Curve Fitting Toolbox and four obtained functions are expressed and used for modeling of pneumatic muscle actuator using modified Hill's muscle model.

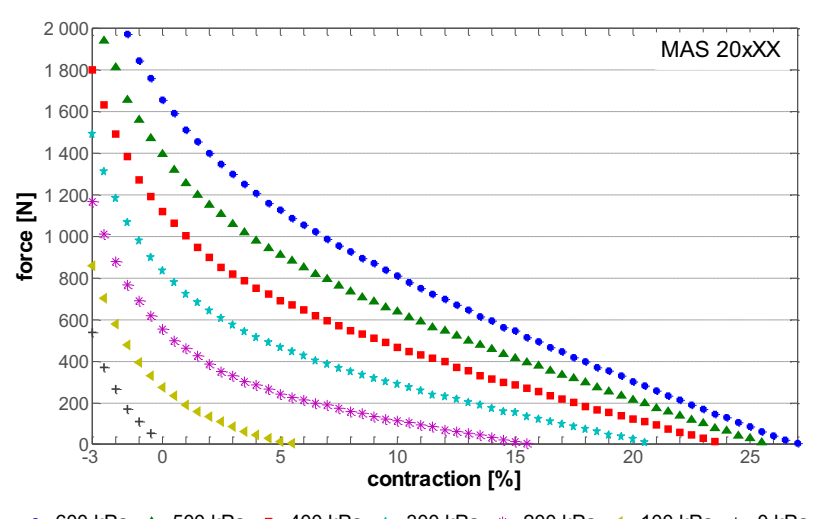

Figure 4. Static characteristics of PAM type FESTO MAS 20200N 12

For practical use, the range of the muscle contraction is limited from $0 \%$ to $25 \%$. At lower muscle contractions, the value of muscle force is already very high and it cause excessive stress materials of the muscle. At higher muscle contractions, there is decrease in the generated force very large. Figure 4 shows that PAM type FESTO MAS 20-20xXX can at the pressure of $600 \mathrm{kPa}$ to exert the muscle force up to $2000 \mathrm{~N}$.

On the basis of physical laws and the geometric parameters of PAM, relation of the static characteristics was derived [7]:

$$
F_{s}(\kappa, p)=\mu(\kappa) \cdot \pi \cdot r_{0}^{2} \cdot p \cdot\left(a \cdot(1-\varepsilon(p) \cdot \kappa)^{2}-b\right)
$$

with member $\mu(\kappa)$ to obtain better approximation for small values of the pressure:

$$
\mu(\kappa)=a_{\kappa} \cdot e^{-\kappa \cdot c_{\kappa}}-b_{\kappa}
$$

and the member $\varepsilon(p)$ to obtain better approximation for higher values of the pressure:

$$
\varepsilon(p)=a_{\varepsilon} \cdot e^{-p}-b_{\varepsilon},
$$

where $p$ is the pressure in the muscle $[\mathrm{Pa}], \kappa$ is the muscle contraction [-], coefficients $a_{\varepsilon}, b_{\varepsilon}, a_{\kappa}, b_{\kappa}, c_{\kappa}$ were determined using Matlab Curve Fitting Toolbox and values $a, b$ have the form [7]:

$$
\begin{gathered}
a=\frac{3}{\tan ^{2} \alpha_{0}}, \\
b=\frac{1}{\sin ^{2} \alpha_{0}},
\end{gathered}
$$


where $\alpha_{0}$ is the initial angle between the muscle axis and its fibers.

The muscle force as a function $F_{s}(\kappa, P)$ of the muscle contraction for different pressures in the muscles can be deducted also from the maximum muscle force $F_{\max }[6]$ :

$$
F_{s}(\kappa, p)=F_{\max }(\kappa)-\left(p_{\max }-p\right) \cdot\left(\frac{a_{0}-a_{1} \kappa}{a_{2}}\right),
$$

where $p_{\max }$ is the maximum and $p$ is the actual pressure in the muscle $[\mathrm{Pa}], \kappa$ is the muscle contraction [-] and coeficients $a_{i}, i=0,1,2$, were found using Matlab Curve Fitting Toolbox [15].

A fourth-order polynomial function for the response at the maximum pressure $p_{\max }=600 \mathrm{kPa}$ was derived for the maximum muscle force $F_{\max }$ and apply [6]:

$$
F_{\max }(\kappa)=b_{0}+b_{1} \kappa+b_{2} \kappa^{2}+b_{3} \kappa^{3}+b_{4} \kappa^{4},
$$

where coeficients $b_{i}, i=1,2,3,4$, were also found using Matlab Curve Fitting Toolbox [15].

The muscle force can be also approximated with a good precision using an exponential function with six different unknown parameters [16]:

$$
\begin{aligned}
F_{s}(\kappa, p) & =\left(a_{1} \cdot p+a_{2}\right) \cdot e^{a_{3} \cdot \kappa}+ \\
& +a_{4} \cdot \kappa \cdot p+a_{5} \cdot p+a_{6},
\end{aligned}
$$

where $\kappa$ is the muscle contraction [-], $p$ is the pressure in the muscle [Pa] and $a_{i}, i=1, . ., 6$ are unknown coefficients which values were also found using Matlab Curve Fitting Toolbox [15].

The fourth method applied for approximation was a polynomial approximation. In order to approximate these static characteristics with good accuracy, a fifthorder polynomial function of two variables was used. This polynomial function contains twenty-one coefficients and its form is as follows [17]:

$$
\begin{aligned}
& F_{s}(\kappa, p)=a_{00}+a_{10} \cdot \kappa+a_{01} \cdot p+a_{20} \cdot \kappa^{2}+ \\
& +a_{11} \cdot \kappa \cdot p+a_{02} \cdot p^{2}+a_{30} \cdot \kappa^{3}+a_{21} \cdot \kappa^{2} \cdot p+ \\
& +a_{12} \cdot \kappa \cdot p^{2}+a_{03} \cdot p^{3}+a_{40} \cdot \kappa^{4}+a_{31} \cdot \kappa^{3} \cdot p+ \\
& +a_{22} \cdot \kappa^{2} \cdot p^{2}+a_{13} \cdot \kappa \cdot p^{3}+a_{04} \cdot p^{4}+a_{50} \cdot \kappa^{5}+ \\
& a_{41} \cdot \kappa^{4} \cdot p+a_{32} \cdot \kappa^{3} \cdot p^{2}+a_{23} \cdot \kappa^{2} \cdot p^{3}+ \\
& +a_{14} \cdot \kappa \cdot p^{4}+a_{05} \cdot p^{5}
\end{aligned}
$$

where $\kappa$ is the muscle contraction [-], $p$ is the pressure in the muscle $[\mathrm{Pa}]$ and the values of other coefficients in (11) were determined also using Matlab Curve Fitting Toolbox [15].

\section{Simulation results}

The designed simulation model of pneumatic muscle actuator based on modified Hill's muscle model was simulated in Matlab/Simulink environment [18] for four different approximations of static force $F_{s}(\kappa, P)$. The simulation results of the actuator arm position for different approximations of static characteristics are shown in Figure 5 and Figure 6. Responses obtained for approximation using an analytical modeling were created using (3), responses obtained for approximation deducted from the maximum force were created using (8) and (9), responses obtained for approximation using an exponential function were created using (10) and responses obtained for approximation using a polynomial function were created using (11).

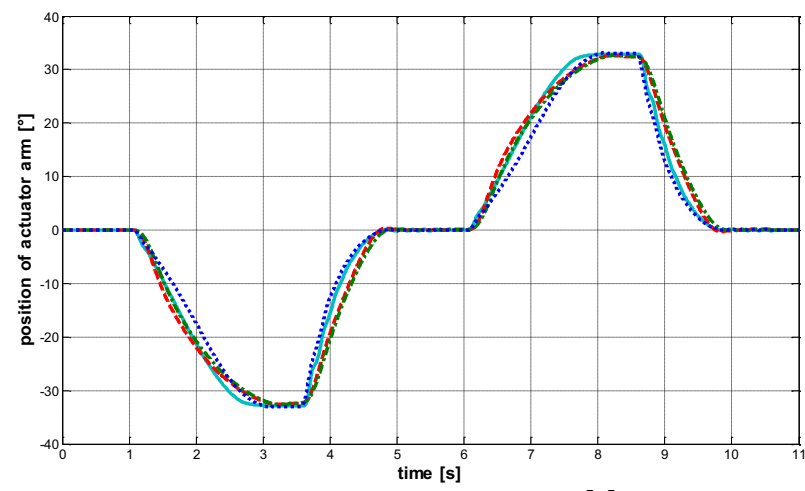

$$
\begin{aligned}
& \text {-Approximation Using an Analytical Modeling of PAM } \\
& \text {-- Approximation Deducted from the Maximum Force of PAM }
\end{aligned}
$$
......Approximation Using a Polynomial Function

Figure 5. Dynamic responses of the actuator arm position for different approximations (maximum angle of actuator arm)

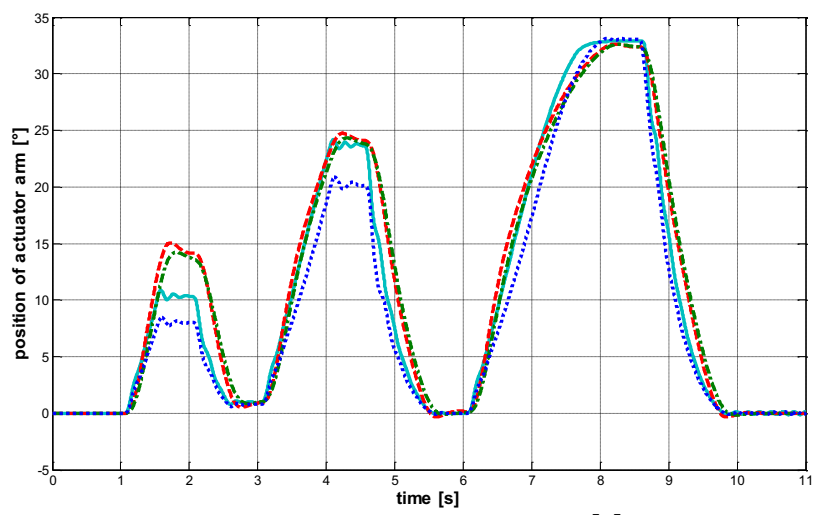

$$
\begin{aligned}
& \text { - Approximation Using an Analytical Modeling of PAM } \\
& \text {---Approximation Deducted from the Maximum Force of PAM } \\
& \text {--.-Approximation Using an Exponential Function } \\
& \text {-... Approximation Using a Polynomial Function }
\end{aligned}
$$

Figure 6. Dynamic responses of the actuator arm position for different approximations (different time periods of filling/discharging air pressure into/from the muscle)

It can be stated, that dynamic responses of the actuator arm position obtained by simulation of pneumatic muscle actuator for approximation deducted from the maximum force and using an exponential function are the similar. On the other hand there are some differences in the simulated responses for used analytical approximation and approximation using polynomial function especially in simulation for different time periods of filling/discharging air pressure into/from the muscle (Figure 6). 


\section{Conclusion}

The muscle force dependence on the muscle contraction and pressure in the muscles was approximated for PAM produced by FESTO Company. Four different approximation functions were tested for possible approximation of the given static characteristics of muscle type MAS-20-200N (Figure 4). Analyses were carried out in Matlab Curve Fitting Toolbox. It can be stated from the obtained simulation results of the pneumatic muscle actuator dynamics using modified Hill's muscle model that the process of approximation of PAM static characteristics has been chosen correctly. The best results were achieved for approximations deducted from the maximum force and using an exponential function and that's why these two approximations are recommended for using in modeling of pneumatic actuators based on artificial muscles. Presented responses are highly non-linear which results high demands on the control algorithms of such actuators.

\section{Acknowledgment}

The research work is supported by grant VEGA 1/0822/16 ,Research of intelligent manipulator based on pneumatic artificial muscles with three degrees of freedom“.

\section{References}

1. J.M. Hopen and A. Hošovský, "The servo robustification of the industrial robot", in Annals of $D A A A M$ for 2005 \& Proceeding $16^{\text {th }}$ International DAAAM Symposium "Intelligent Manufacturing \& Automation: Focus on Young Scientists and Researchers", Vienna, 2005. pp. 161-162 (2005)

2. O. Líška, M. More, D. Janáčová, and H. Charvátová, "Design of rehabilitation robot based on pneumatic artificial muscles", in Proceedings of Mathematical Methods and Optimization Techniques in Engineering, Antalya, 2013. pp. 151-154 (2013)

3. A. Hošovský and M. Havran, "Dynamic modeling of one degree of freedom pneumatic muscle-based actuator for industrial applications", Tehnički Vjesnik - Technical Gazette, Vol. 19, No. 3, 2012. pp. 73-681 (2012)

4. A. Hošovský and M. Havran, "Hill's muscle modelbased modeling of pneumatic artificial muscle", in Annals of DAAAM for 2011 \& Proceedings of the 22nd International DAAAM Symposium, Vienna, 2011. pp. 1005-1006 (2011)

5. J. Boržíková, "The determination of analytic dependence of static characteristic of PAM-based antagonistic actuator", Acta Mechanica Slovaca, Vol. 12, No. 1-A, 2008. pp. 227-230. (2008)

6. V.T. Jouppila, S.A. Gadsden, and A. Ellman, "Modeling and identification of a pneumatic muscle actuator system controlled by an ON/OFF solenoid valve", in Proceeding 7th International Fluid Power Conference, Aachen, 2010, pp. 1-11 (2010)
7. T. Kerscher, J. Albiez, J.M. Zollner, and R. Dillmann, "Evaluation of the dynamic model of fluidic muscles using quick-release", in Proceeding of International Conference on Biomedical Robotics and Biomechatronics (BioRob 2006), Pisa, 2006. pp. 637-642 (2006)

8. M. Tóthová, and J. Pitel', "Dynamic simulation of pneumatic muscle actuator in Matlab/Simulink environment", in Proceedings of the IEEE 12th International Symposium on Intelligent Systems and Informatics (SISY 2014), Subotica, 2014. pp. 209213. (2014)

9. J. Sárosi, I. Biro, L. Nemeth, and I. Cveticanin, "Dynamic modeling of a pneumatic muscle actuator with two-direction motion", Mechanism and Machine Theory, Vol. 85, 2015. pp. 25-34. (2015)

10. M. Tóthová and J. Pitel', "Simulation of actuator dynamics based on geometric model of pneumatic artificial muscle", in Proceedings of the IEEE 11th International Symposium on Intelligent Systems and Informatics (SISY 2013), Subotica, 2013. pp. 233237 (2013)

11. M. Tóthová and J. Pitel', Dynamic model of pneumatic actuator based on advanced geometric muscle model", in Proceedings of the IEEE 9th International Conference on Computational Cybernetics (ICCC 2013), Budapest, 2013. pp. 83-87 (2013)

12. J. Pitel', M. Balara, A. Hošovský, and M. Tothova, Pneumatic muscle actuators: modelling, simulation and control (Košice: TU, 275 p. 2015)

13. J. Boržíková, "Non-linear approximation of the static characteristic $\mathrm{F}=\mathrm{f}(\mathrm{p}, \kappa)$ of antagonistic system", in Proceeding ARTEP 2008, Stará Lesná, 2008. pp. 41-5 (2008)

14. Fluidic muscles DMSP-../MAS-... [online] Available at: https://www.festo.com/cat/enus_us/data/doc_enus/PDF/US/DMSPMĀS_ENUS.PDF (2016)

15. M. Tóthová, J. Pitel', A. Hošovský, and J. Sárosi, "Numerical approximation of static characteristics of McKibben pneumatic artificial muscle", International Journal of Mathematics and Computers in Simulation, Vol. 9, 2015. pp. 228-233 (2015)

16. J. Sárosi, "New approximation algorithm for the force of fluidic muscles", in Proceeding $7^{\text {th }}$ International Symposium on Applied Computational Intelligence and Informatics (SACI 2012), Timisoara, 2012, pp. 229-233 (2012)

17. A. Hošovský, Methods of computational intelligence in the control and modeling of manipulators driven by artificial muscles (Habilitation work, Prešov: TU, 135 p. 2014)

18. M. Tóthová and A. Hošovský, "Dynamic simulation model of pneumatic actuator with artificial muscle", in Proceedings of the 11th IEEE International Symposium on Applied Machine Intelligence and Informatics (SAMI 2013), Budapest, 2013. pp. 47-51 (2013) 